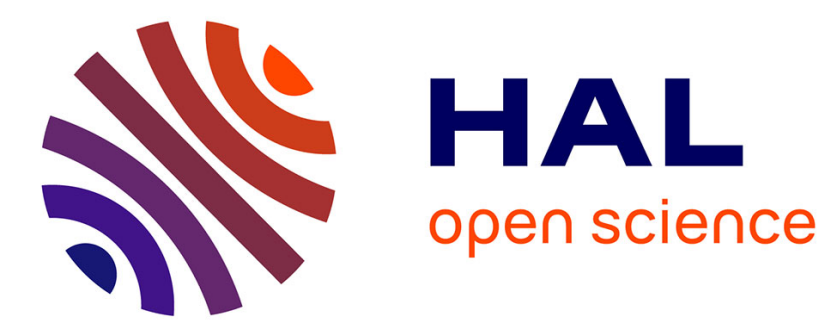

\title{
Qualitative stability patterns for Lotka-Volterra systems on rectangles
}

\author{
Laurent Tournier, Jean-Luc Gouzé
}

\section{To cite this version:}

Laurent Tournier, Jean-Luc Gouzé. Qualitative stability patterns for Lotka-Volterra systems on rectangles. [Research Report] RR-6346, INRIA. 2007, pp.16. inria-00186247v2

\section{HAL Id: inria-00186247 https://hal.inria.fr/inria-00186247v2}

Submitted on 8 Nov 2007

HAL is a multi-disciplinary open access archive for the deposit and dissemination of scientific research documents, whether they are published or not. The documents may come from teaching and research institutions in France or abroad, or from public or private research centers.
L'archive ouverte pluridisciplinaire HAL, est destinée au dépôt et à la diffusion de documents scientifiques de niveau recherche, publiés ou non, émanant des établissements d'enseignement et de recherche français ou étrangers, des laboratoires publics ou privés. 
INSTITUT NATIONAL DE RECHERCHE EN INFORMATIQUE ET EN AUTOMATIQUE

\section{Qualitative stability patterns for Lotka-Volterra systems on rectangles}

Laurent Tournier — Jean-Luc Gouzé

\section{$\mathrm{N}^{\circ} 6346$}

November 2007

Thème $\mathrm{BIO}$

apport

derecherche 



\title{
Qualitative stability patterns for Lotka-Volterra systems on rectangles
}

\author{
Laurent Tournier*, Jean-Luc Gouzé ${ }^{\dagger}$ \\ Thème BIO — Systèmes biologiques \\ Projet Comore \\ Rapport de recherche $\mathrm{n}^{\circ} 6346$ - November 2007 - 16 pages
}

\begin{abstract}
We present a qualitative analysis of the Lotka-Volterra differential equation within rectangles that are transverse with respect to the flow. In similar way to existing works on affine systems (and positively invariant rectangles), we consider here nonlinear Lotka-Volterra $n$-dimensional equation, in rectangles with any kind of tranverse patterns. We give necessary and sufficient conditions for the existence of symmetrically transverse rectangles (containing the positive equilibrium), giving notably the method to build such rectangles. We also analyse the stability of the equilibrium thanks to this transverse pattern. We finally propose an analysis of the dynamical behavior inside a rectangle containing the positive equilibrium, based on Lyapunov stability theory. More particularly, we make use of Lyapunov-like functions, built upon vector norms. This work is a first step towards a qualitative abstraction and simulation of Lotka-Volterra systems.
\end{abstract}

Key-words: Dynamical systems, Lotka-Volterra equation, Transverse rectangle, Qualitative analysis, Vector norms Lyapunov functions.

* Laurent.Tournier@sophia.inria.fr

† gouze@sophia.inria.fr 


\section{Analyse qualitative des systèmes de Lotka-Volterra sur des rectangles}

Résumé : Ce travail pose les bases d'une étude qualitative des systèmes dynamiques de LotkaVolterra, fondée sur l'étude de rectangles transverses par rapport au flot. Comparativement à de précédentes études similaires, nous nous intéressons ici à des rectangles non nécessairement positivement invariants, et les résultats présentés ne se limitent pas au cas linéaire mais sont généralisés au cas non linéaire (de dimension $n$ ) de Lotka-Volterra. Nous donnons notamment des conditions nécessaires et suffisantes pour l'existence de rectangles symétriquement transverses (qui donc contiennent l'équilibre positif) en proposant une méthode pour les construire. Ces conditions nous permettent en outre de déduire du patron de ces rectangles le type de stabilité de l'équilibre. Nous proposons enfin une analyse de la dynamique du système à l'intérieur de ces rectangles, en utilisant la théorie générale de stabilité de Lyapunov. Les fonctions de type Lyapunov que nous construisons ici utilisent des normes vectorielles. Ce travail doit être vu comme une première étape vers une analyse qualitative de la dynamique de l'équation non linéaire de Lotka-Volterra.

Mots-clés : Systèmes dynamiques, Equation de Lotka-Volterra, Rectangle transverse au flot, Analyse qualitative, Fonctions de Lyapunov basées sur des normes vectorielles. 


\section{Introduction}

We consider in this paper $n$-dimensional nonlinear Lotka-Volterra (LV) differential systems in $n$-dimensional rectangular regions of the phase space. This work comes within the context of hybrid analysis of nonlinear dynamical systems. In classical hybrid approach, piecewise linear or affine systems are used in simplexes, rectangles or more general polytopes (see for instance [4] or [7]). More recently, [11] proposed a more general multi-affine framework. Because of its square terms, Lotka-Volterra equation does not properly enter this class of differential equations. It is nevertheless a quite general class of nonlinear dynamical systems, widely used in biology and especially in ecology and population dynamics models (see notably [8]).

We present some theoretical results about these systems, and notably a necessary and sufficient condition on the matrix of the system for the existence of a $n$-dimensional rectangle, containing the equilibrium, and which is transverse with respect to the flow. We also give a method to construct such rectangles. It is to be related with [1] (and $[3,5]$ for the case of general polytopes) in which similar studies are done in the linear framework, in the case where the rectangles are transverse and positively invariant. As LV systems are nonlinear, we finally propose a global analysis of the dynamics inside transverse rectangles, based on Lyapunov stability theory.

Proposition 2 and theorem 4 can be seen as a first step towards a qualitative analysis of LV systems. Using a rectangular mesh of the phase space, one can indeed qualitatively describe sets of continuous trajectories by giving a sequence of transitions between rectangular regions. Besides the algorithmic power of such an abstraction approach (see [4] in a different framework), it has the advantage of being particularly robust with respect to the parameters (in a lot of applications, the value of certain parameters are indeed only loosely known).

Throughout this paper, we will denote $\leq$ and $\ll$ the classical large and strict partial ordering of $\mathbb{R}^{n}$ : given $x, y \in \mathbb{R}^{n}, x \leq y$ (respectively $x \ll y$ ) iff $x_{i} \leq y_{i}$ (resp. $x_{i}<y_{i}$ ) for each $i=1, \ldots, n$. The symbol $\otimes$ designates the component-wise product of real vectors. Symbols $\ln (x)$ and $|x|$, when $x$ is a $n$-dimensional real vector denote the vectors $\left(\ln \left(x_{1}\right), \ldots, \ln \left(x_{n}\right)\right)$ and $\left(\left|x_{1}\right|, \ldots,\left|x_{n}\right|\right)$

We consider the LV $n$-dimensional dynamical system (see [8]):

$$
\dot{x}(t)=x(t) \otimes(A x(t)+b)
$$

where $x(t)$ lies in $\mathbb{R}^{n}, A$ is a constant $n \times n$ real matrix and $b$ is a fixed vector in $\mathbb{R}^{n}$. In order to focus on nondegenerate cases, we will suppose throughout this paper that the matrix $A$ is invertible. We are then ensured of the existence and uniqueness of the non-zero equilibrium: $x^{*}=-A^{-1} b$. It is well known that the hyperplanes $x_{i}=0$ are invariant, and so is each orthant, delimited by these hyperplanes. For sake of clarity, we will suppose that $x^{*} \gg 0$, and we will study this system in the positive orthant:

$$
\dot{x}=x \otimes A\left(x-x^{*}\right)=: f^{\mathrm{lv}}(x), \quad x \in\left(\mathbb{R}_{+}^{*}\right)^{n}
$$

Thanks to its mathematical structure, (1) shares some properties with the associate simple affine system:

$$
\dot{x}=A\left(x-x^{*}\right)=: f^{\text {lin }}(x), \quad x \in \mathbb{R}^{n}
$$

In particular, the results presented in this article are actually valid in both frameworks (provided we limit ourselves to the positive orthant). However it is to be recalled that, despite that fact, these two systems are dynamically very different. Indeed, due to its nonlinearity, LV system (1) can exhibit complex asymptotic behaviors such as heteroclinic limit cycles or chaotic attractors (when the dimension $n \geq 3$ ), whereas affine system (2) cannot.

\section{Rectangles with fixed transverse patterns}

Throughout this paper, we are interested in the dynamical behavior of systems (1) and (2) in full-dimensional straight rectangles, defined as follows:

Definition 1 Let $m, M$ be two $n$-dimensional real vectors satisfying $m \ll M$. The full-dimensional rectangle delimited by $m$ and $M$, denoted $[m, M]$ is the set $[m, M]=\left\{x \in \mathbb{R}^{n} \mid m \leq x \leq M\right\}$.

$\mathrm{RR} \mathrm{n}^{\circ} 6346$ 
These rectangles are polytopes, ie bounded polyhedral sets (see [14] for a proper definition), that have exactly $2^{n}$ vertices and $2 n$ faces of dimension $n-1$. Let $R$ denote the rectangle $[m, M]$, $\Sigma_{R}$ the set of its vertices. The latter are points of $\mathbb{R}^{n}$ that can be indexed by a boolean vector $v \in\{0,1\}^{n}$ as follows:

$$
\Sigma_{R}=\left\{\sigma^{v} \mid v \in\{0,1\}^{n}\right\} \text { with, for } i \in\{1, \ldots, n\},\left(\sigma^{v}\right)_{i}= \begin{cases}m_{i}, & \text { if } v_{i}=0 \\ M_{i}, & \text { if } v_{i}=1\end{cases}
$$

Let us consider now the faces of $R$ of dimension $n-1$. In each direction $i \in\{1, \ldots, n\}, R$ admits two faces, noted $\mathcal{F}_{i}^{+}$and $\mathcal{F}_{i}^{-}$and defined by:

$$
\begin{aligned}
\mathcal{F}_{i}^{+} & =\prod_{j=1}^{i-1}\left[m_{j}, M_{j}\right] \times\left\{M_{i}\right\} \times \prod_{j=i+1}^{n}\left[m_{j}, M_{j}\right] \\
\mathcal{F}_{i}^{-} & =\prod_{j=1}^{i-1}\left[m_{j}, M_{j}\right] \times\left\{m_{i}\right\} \times \prod_{j=i+1}^{n}\left[m_{j}, M_{j}\right]
\end{aligned}
$$

These are rectangles of dimension $n-1$ according to definition 1 . They have $2^{n-1}$ vertices, that can be expressed in function of the vertices of $R: \Sigma_{\mathcal{F}_{i}^{+}}=\left\{\sigma^{v} \mid v \in\{0,1\}^{n}, v_{i}=1\right\}$ and $\Sigma_{\mathcal{F}_{i}^{-}}=\left\{\sigma^{v} \mid v \in\{0,1\}^{n}, v_{i}=0\right\}$. It is easy to see that these two sets are disjoint and we have: $\Sigma_{R}=\Sigma_{\mathcal{F}_{i}^{+}} \cup \Sigma_{\mathcal{F}_{i}^{-}}$. Figure 1 shows an illustration of these notations in two dimensions.

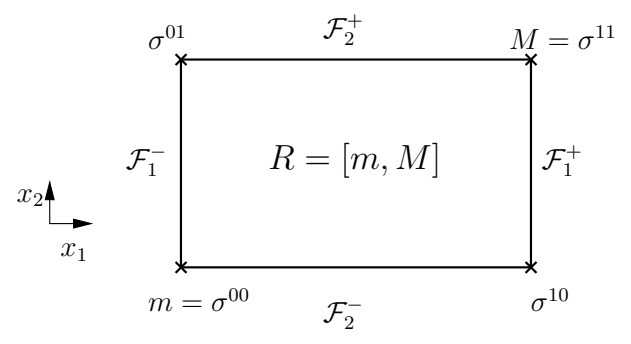

Figure 1: A bidimensional straight rectangle.

We now give a proper definition of the word transverse that we have used so far.

Definition 2 Let $m \ll M$ be two real vectors and $R=[m, M] ; f$ designates a continuous vector field $f: R \rightarrow \mathbb{R}^{n}$. Given $i \in\{1, \ldots, n\}$ and $\varepsilon \in\{+,-\}$, we say that the face $\mathcal{F}_{i}^{\varepsilon}$ of $R$ is transverse with respect to $f$ if $f_{i}$ does not vanish on $\mathcal{F}_{i}^{\varepsilon}$ and if $f_{i}(x)$, for $x$ varying in $\mathcal{F}_{i}^{\varepsilon}$, keeps a constant sign $s \in\{+,-\}$.

- if $s \neq \varepsilon$ the face $\mathcal{F}_{i}^{\varepsilon}$ is incoming (the field points inward $R$ ),

- if $s=\varepsilon$ the face $\mathcal{F}_{i}^{\varepsilon}$ is outgoing (the field points outward $R$ ).

We will say that the rectangle $R$ has a fixed transverse pattern if its $2 n$ faces are transverse. Furthermore, we will say that $R$ has a symmetrical pattern if it also satisfies the following property: for all $i \in\{1, \ldots, n\}$, the faces $\mathcal{F}_{i}^{-}$and $\mathcal{F}_{i}^{+}$are either both incoming or both outgoing.

Fig. 2 shows several examples of rectangles with fixed transverse patterns, symmetrical or not. In fig. $2 \mathrm{~d}$, all edges are incoming, $R$ is then a positively invariant set. This is the most studied case in the literature (see $[1,3]$ ).

As we said, rectangles are specific kind of polytopes, and as such they satisfy several well-known properties. One of this property is the following lemma (based on the convexity of polytopes, see notably [14]):

Lemma 1 Let $R$ be a rectangle of any dimension in $\mathbb{R}^{n}$, and let $\phi$ be a linear application $\phi: \mathbb{R}^{n} \rightarrow$ $\mathbb{R}^{p}$, for $p \in \mathbb{N}^{*}$. Then ${ }^{1}, \phi(R)=\operatorname{co}\left(\left\{\phi(\sigma) \mid \sigma \in \Sigma_{R}\right\}\right) \subset \mathbb{R}^{p}$.

\footnotetext{
${ }^{1}$ The notation $\overline{\mathrm{CO}}(X)$ designates the closed convex hull of the set $X$
} 
Considering affine and LV vector fields, one can easily show that the transversality of a face can be checked directly from the signs of the $f_{i}(\sigma)$, where the $\sigma$ are the vertices of the face:

Proposition 1 Let $0 \ll m \ll M$ and $R=[m, M], f$ designates either the affine or the LV vector field. For each $i \in\{1, \ldots, n\}$ and $\varepsilon \in\{+,-\}$, the face $\mathcal{F}_{i}^{\varepsilon}$ is transverse if and only if, for all $\sigma \in \Sigma_{\mathcal{F}_{i}^{\varepsilon}}$, the real quantities $A_{i} \cdot\left(\sigma-x^{*}\right)$ are not zero ${ }^{2}$ and share the same sign $s \in\{-,+\}$. If this property is satisfied, then, if $s \neq \varepsilon$ the face $\mathcal{F}_{i}^{\varepsilon}$ is incoming, otherwise it is outgoing.

\section{Proof}

In the case of affine vector field, this result is an immediate consequence of lemma 1. For LV vector field, we just remark that, for each $i \in\{1, \ldots, n\}$ :

$$
f_{i}^{\mathrm{lv}}(x)=x_{i}\left(A_{i}\left(x-x^{*}\right)\right)=x_{i} f_{i}^{\operatorname{lin}}(x)
$$

Since $x \geq m \gg 0$, the quantities $f_{i}^{\mathrm{lv}}(x)$ and $f_{i}^{\text {lin }}(x)$ vanish exactly at the same points and share the same sign.

What we propose in this paper is an analysis of the dynamics of systems (1) and (2) in rectangles with fixed transverse patterns. Such a study has been performed for linear systems in the case where the rectangle is stricly positively invariant, such as in fig. $2 \mathrm{~d}$ (see for instance [1], and [3] for a similar study for general invariant polytopes). We generalize these results for a rectangle with any symmetrical transverse pattern. We also propose a generalization to the Lotka-Volterra framework, following a precedent work [6]. We first give the following

Proposition 2 Let $0 \ll m \ll M$ and $R=[m, M]$, $f$ designates the affine or LV vector field. Then,

(i) Suppose that $R$ is transverse. Then, $x^{*} \in R$ if and only if the pattern of $R$ is symmetrical (and then $x^{*}$ belongs necessarily to the interior of $R$ ).

(ii) Suppose $x^{*} \notin R$. Then, given any initial condition $x^{0} \in R$, the solution $t \mapsto x\left(t, x^{0}\right)$ of $\dot{x}=f(x)$ leaves $R$ in finite time.

\section{Proof}

Let $R$ have a fixed transverse pattern. Suppose that the pattern is not symmetrical, then there exists $i \in\{1, \ldots, n\}$ such that $\mathcal{F}_{i}^{+}$and $\mathcal{F}_{i}^{-}$are not symmetrical. Using (3) and proposition 1 , we deduce that:

$$
\left(\forall v \in\{0,1\}^{n}, f_{i}^{\operatorname{lin}}\left(\sigma^{v}\right)>0\right) \text { or }\left(\forall v \in\{0,1\}^{n}, f_{i}^{\operatorname{lin}}\left(\sigma^{v}\right)<0\right)
$$

Thanks to lemma $1, f_{i}^{\text {lin }}$ is therefore either positive or negative on $R$ which implies that $x^{*} \notin R$. Conversely, suppose that the pattern is symmetrical. Let $\mathcal{P}$ be the convex hull of the points $\left\{f^{\operatorname{lin}}\left(\sigma^{v}\right) \mid \sigma^{v} \in \Sigma_{R}\right\}$. By definition, $\mathcal{P}$ is a polytope with at least $2^{n}$ vertices. As each face of $R$ is transverse, for each $\sigma^{v} \in \Sigma_{R}$ and each $i \in\{1, \ldots, n\}$, the quantity $f_{i}^{\text {lin }}\left(\sigma^{v}\right)$ is non zero, and therefore $f^{\operatorname{lin}}\left(\sigma^{v}\right)$ lies in an open orthant of $\mathbb{R}^{n}$. In the following, $\mathcal{O}_{v}$ denotes the open orthant of $\mathbb{R}^{n}$ equals to $\left\{x \in \mathbb{R}^{n} \mid(-1)^{v_{i}} x_{i}>0, i=1 \ldots n\right\}$. We consider the map $\Phi:\{0,1\}^{n} \rightarrow\{0,1\}^{n}$ such that for all $v \in\{0,1\}^{n}, f^{\text {lin }}\left(\sigma^{v}\right)$ belongs to the orthant $\mathcal{O}_{\Phi(v)}$. Let us show that $\Phi$ is bijective.

Let $v, v^{\prime} \in\{0,1\}^{n}, v \neq v^{\prime}$. There is $i \in\{1, \ldots, n\}$ such that $v_{i} \neq v_{i}^{\prime}$, for instance $v_{i}=0$ and $v_{i}^{\prime}=1$. We have then $\sigma^{v} \in \mathcal{F}_{i}^{-}$and $\sigma^{v^{\prime}} \in \mathcal{F}_{i}^{+}$. Since the pattern is symmetrical, we have:

$$
\operatorname{sgn}\left(f_{i}^{\operatorname{lin}}\left(\sigma^{v}\right)\right) \neq \operatorname{sgn}\left(f_{i}^{\operatorname{lin}}\left(\sigma^{v^{\prime}}\right)\right)
$$

so $f^{\text {lin }}\left(\sigma^{v}\right)$ and $f^{\text {lin }}\left(\sigma^{v^{\prime}}\right)$ do not belong to the same orthant and thus $\Phi(v) \neq \Phi\left(v^{\prime}\right)$. $\Phi$ is injective and as it maps $\{0,1\}^{n}$ to itself, it is a bijection. We deduce from this that each vertex of the polytope $\mathcal{P}$ lies in a different orthant of $\mathbb{R}^{n}$, and since $\mathcal{P}$ is convex, this implies that $0 \in \mathcal{P}$. Therefore, thanks to lemma 1 , there exists $y \in R$ verifying $f^{\operatorname{lin}}(y)=0$. By definition, we also have that $f^{\operatorname{lv}}(y)=y \otimes f^{\operatorname{lin}}(y)=0$. Thanks to the uniqueness of the equilibrium $x^{*}$ (in both linear and

\footnotetext{
${ }^{2} A_{i}$ designates the $i$ th line of matrix $A$ (it is so a row vector)
} 
Lotka-Volterra case), we get that $y=x^{*}$ and finally that $x^{*} \in R$ (necessarily, $x^{*}$ belongs to the interior of $R$ as all the faces of $R$ are transverse).

The proof of point (ii) is quite classical (see for instance [8]) and relies on the well-known separating hyperplane theorem. Indeed, if $R$ has a non-symmetrical pattern, then $x^{*} \notin R$ and as $R$ is convex, there exists a non-zero vector $h \in \mathbb{R}^{n}$ such that:

$$
\forall x \in R, \quad h^{t} \cdot\left(x-x^{*}\right)>0
$$

and such that $h^{t} \cdot x^{*}>0$. We thus have, as $R$ is compact: $\inf _{x \in R} h^{t} \cdot x=c>0$. It then suffices to find a function $V: R \rightarrow \mathbb{R}$, of class $\mathcal{C}^{1}$ and such that $\dot{V}(x)=h^{t} \cdot x>0$ (a classical result in dynamical system ensures then that the compact $R$ is left in finite time). For linear system, we choose $V^{\operatorname{lin}}(x)=q^{t} \cdot x$ with $q^{t}=h^{t} \cdot A^{-1}$. For LV system, it suffices to choose $V^{\mathrm{lv}}(x)=q^{t} \cdot \ln (x)$ with the same vector $q$.

Remark 1 Concerning the fact that the symmetry of the transverse pattern of $R$ implies that the equilibrium $x^{*}$ belongs to $R$, the proof that is given here is essentially geometrical, and is based on the fact that $R$ is a polytope. It can be seen however from a different point of view. Indeed, one can prove it using a well-known result in ODE theory: for a smooth dynamical system, any nonempty strictly positively invariant set $X$ (homeomorphic to a ball of $\mathbb{R}^{n}$ ) contains an equilibrium point. This property can be found for instance in [8], and mainly relies on the Brouwer fixed-point theorem. To apply it in our case, we denote by $I$ the set of the $i \in\{1, \ldots, n\}$ such that the faces $\mathcal{F}_{i}^{ \pm}$are outgoing, and we define the vector field $\widetilde{f}$ as follows:

$$
\forall i \in I, \widetilde{f}_{i}(x)=-f_{i}^{l i n}(x) \quad \text { and } \quad \forall i \in\{1, \ldots, n\} \backslash I, \widetilde{f}_{i}(x)=f_{i}^{l i n}(x)
$$

It is easy to see that flin and $\tilde{f}$ have exactly the same equilibria, and that $R$ is strictly positively invariant for $\widetilde{f}$, which leads us to the wanted result.

We deduce from this proposition that any rectangle $R$ that does not contain the equilibrium is transient (in the sense that any trajectory starting in $R$ eventually leaves $R$ ), regardless of the fact that it is transverse. As we will see in part 4, the transversality is however important in order to describe the dynamics inside the rectangle containing the equilibrium. We therefore start by giving some conditions to ensure that this particular rectangle is transverse (let us note that in that case, according to proposition 2, it has a symmetrical pattern).
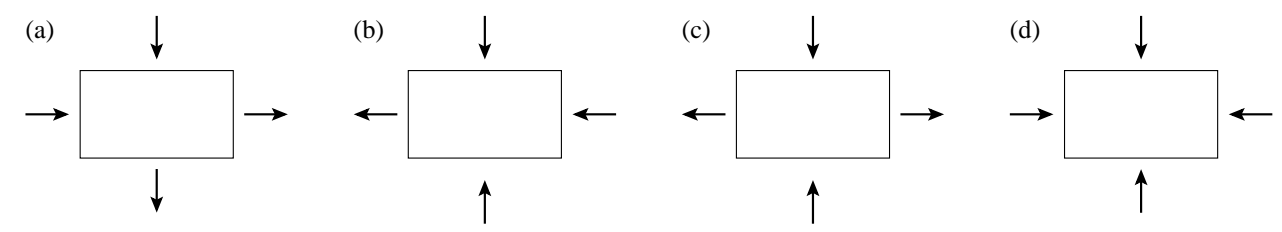

Figure 2: Examples of 2-dimensional rectangles with fixed transverse patterns. (c) and (d) have symmetrical patterns.

\section{Necessary and sufficient conditions for the existence of a transverse rectangle, with symmetrical pattern}

The topic of this part is to link the existence of a transverse rectangle (with a symmetrical pattern) with the structure of the matrix $A$. We will mainly use in this part some classical theorems about matrices that can be found in $[2,9]$. A part of the results exposed here are already known, in the case of linear systems and positively invariant rectangles [1] (see also [3,5] in the case of more general polytopes). We propose here a general result with any kind of symmetrical pattern. We moreover develop the converse, and we notably give an explicit method to build transverse rectangles (with 
the wanted pattern) which are not necessarily symmetrical (in a geometrical sense) around the equilibrium.

We adopt the following notation (see [2]): $Z^{n \times n}=\left\{A \in \mathbb{R}^{n \times n} \mid \forall i \neq j, a_{i j} \leq 0\right\}$. A well-known and useful subclass of this set of matrices is the class of $M$-matrices, defined in [2]. The main interest of this class of real matrices lies in the very complete theorem that can be found in $[2, \mathrm{p}$ 134], giving characterizations for a matrix $A \in Z^{n \times n}$ to be a non-singular M-matrix. We finally recall the following classical definition $[2,9]$ :

Definition 3 Let $A=\left(a_{i j}\right)$ be a real $n \times n$ matrix. The comparison matrix of $A$, noted $\mathcal{M}(A)=$ $\left(m_{i j}\right)$, is the matrix of $Z^{n \times n}$ defined by:

- For $i \in\{1, \ldots, n\}, m_{i i}=\left|a_{i i}\right|$,

- for $i, j \in\{1, \ldots, n\}, i \neq j, m_{i j}=-\left|a_{i j}\right|$.

As we said in previous sections, the transverse pattern of a rectangle $R=[m, M]$, provided we impose $m \gg 0$, is exactly the same for linear system (2) and LV system (1). We will so consider in this part the generic system:

$$
\dot{x}=f(x)
$$

where $f$ designates either: $f(x)=A\left(x-x^{*}\right)$ or $f(x)=x \otimes A\left(x-x^{*}\right)$. For all $(i, j) \in\{1, \ldots, n\}^{2}$, we use the following notations: $a_{i j}^{+}=\max \left(a_{i j}, 0\right)$ and $a_{i j}^{-}=\min \left(a_{i j}, 0\right)$. We therefore have: $a_{i j}^{+}+a_{i j}^{-}=a_{i j}$ and $a_{i j}^{+}-a_{i j}^{-}=\left|a_{i j}\right|$. We will note $A^{+}$(resp. $A^{-}$) the $n \times n$ matrix of the $a_{i j}^{+}$(resp. $\left.a_{i j}^{-}\right)$with a zero diagonal.

\subsection{Necessary conditions and stability analysis}

We suppose in this part the existence of a $n$-dimensional rectangle $R=[m, M]$ (with $m, M \in \mathbb{R}^{n}$, $0 \ll m \ll M)$, that admits a symmetrical transverse pattern for system (5), with $p \in\{0, \ldots, n\}$ pairs of outgoing faces and $n-p$ pairs of incoming faces. In order to lighten the notations, we can suppose (possibly reordering the variables) that for $i=1, \ldots, p, \mathcal{F}_{i}^{+}$and $\mathcal{F}_{i}^{-}$are outgoing, and for $i=p+1, \ldots, n, \mathcal{F}_{i}^{+}$and $\mathcal{F}_{i}^{-}$are incoming, $i e$, according to proposition $1\left(A_{i}\right.$ denoting the $i$ th row of $A$ ):

$$
i=1 . . p:\left\{\begin{array}{l}
\forall x \in \mathcal{F}_{i}^{-}, A_{i} \cdot\left(x-x^{*}\right)<0 \\
\forall x \in \mathcal{F}_{i}^{+}, A_{i} .\left(x-x^{*}\right)>0
\end{array} \quad i=p+1 . . n:\left\{\begin{array}{l}
\forall x \in \mathcal{F}_{i}^{-}, A_{i} \cdot\left(x-x^{*}\right)>0 \\
\forall x \in \mathcal{F}_{i}^{+}, A_{i} \cdot\left(x-x^{*}\right)<0
\end{array}\right.\right.
$$

A 3-dimensional example can be found in fig. 3. We recall that according to proposition 2, this implies that the equilibrium $x^{*}$ lies in the interior of the rectangle $R$.

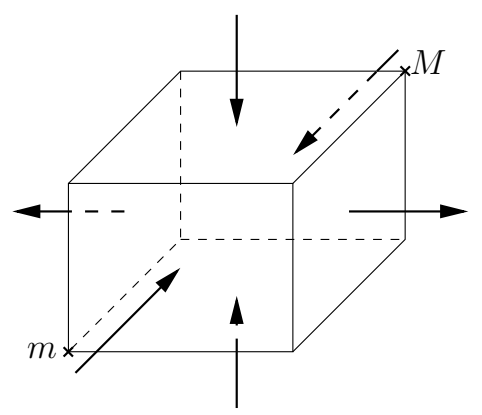

Figure 3: A 3-dimensional rectangle with a symmetrical transverse pattern. The faces $\mathcal{F}_{1}^{ \pm}$are outgoing and the other faces are incoming (ie $p=1$ ). 
Lemma 2 For all $i \in\{1, \ldots, n\}$, we have:

$$
\begin{aligned}
\min _{x \in \mathcal{F}_{i}^{-}}\left[A_{i} \cdot\left(x-x^{*}\right)\right] & =a_{i i}\left(m_{i}-x_{i}^{*}\right)+\sum_{j \neq i}\left[a_{i j}^{+}\left(m_{j}-x_{j}^{*}\right)+a_{i j}^{-}\left(M_{j}-x_{j}^{*}\right)\right] \\
\max _{x \in \mathcal{F}_{i}^{-}}\left[A_{i} \cdot\left(x-x^{*}\right)\right] & =a_{i i}\left(m_{i}-x_{i}^{*}\right)+\sum_{j \neq i}\left[a_{i j}^{+}\left(M_{j}-x_{j}^{*}\right)+a_{i j}^{-}\left(m_{j}-x_{j}^{*}\right)\right] \\
\min _{x \in \mathcal{F}_{i}^{+}}\left[A_{i} \cdot\left(x-x^{*}\right)\right] & =a_{i i}\left(M_{i}-x_{i}^{*}\right)+\sum_{j \neq i}^{j}\left[a_{i j}^{+}\left(m_{j}-x_{j}^{*}\right)+a_{i j}^{-}\left(M_{j}-x_{j}^{*}\right)\right] \\
\max _{x \in \mathcal{F}_{i}^{+}}\left[A_{i} \cdot\left(x-x^{*}\right)\right] & =a_{i i}\left(M_{i}-x_{i}^{*}\right)+\sum_{j \neq i}\left[a_{i j}^{+}\left(M_{j}-x_{j}^{*}\right)+a_{i j}^{-}\left(m_{j}-x_{j}^{*}\right)\right]
\end{aligned}
$$

This lemma directly comes from our notations, and its proof is left to the reader. Thanks to lemma 2, system (6) is equivalent to the following:

$$
\begin{gathered}
\forall 1 \leq i \leq p:\left\{\begin{array}{l}
a_{i i}\left(m_{i}-x_{i}^{*}\right)+\sum_{j \neq i}\left[a_{i j}^{+}\left(M_{j}-x_{j}^{*}\right)+a_{i j}^{-}\left(m_{j}-x_{j}^{*}\right)\right]<0 \\
a_{i i}\left(M_{i}-x_{i}^{*}\right)+\sum_{j \neq i}\left[a_{i j}^{+}\left(m_{j}-x_{j}^{*}\right)+a_{i j}^{-}\left(M_{j}-x_{j}^{*}\right)\right]>0
\end{array}\right. \\
\forall p+1 \leq i \leq n:\left\{\begin{array}{l}
a_{i i}\left(m_{i}-x_{i}^{*}\right)+\sum_{j \neq i}\left[a_{i j}^{+}\left(m_{j}-x_{j}^{*}\right)+a_{i j}^{-}\left(M_{j}-x_{j}^{*}\right)\right]>0 \\
a_{i i}\left(M_{i}-x_{i}^{*}\right)+\sum_{j \neq i}\left[a_{i j}^{+}\left(M_{j}-x_{j}^{*}\right)+a_{i j}^{-}\left(m_{j}-x_{j}^{*}\right)\right]<0
\end{array}\right.
\end{gathered}
$$

By substracting the two inequations of the two systems, we obtain:

$$
\begin{aligned}
\forall 1 \leq i \leq p: & a_{i i}\left(M_{i}-m_{i}\right)+\sum_{j \neq i}\left(-\left|a_{i j}\right|\right)\left(M_{j}-m_{j}\right)>0 \\
\forall p+1 \leq i \leq n: & a_{i i}\left(M_{i}-m_{i}\right)+\sum_{j \neq i}\left|a_{i j}\right|\left(M_{j}-m_{j}\right)<0
\end{aligned}
$$

In order to facilitate the expression of these inequalities, we define the following block matrix and vector:

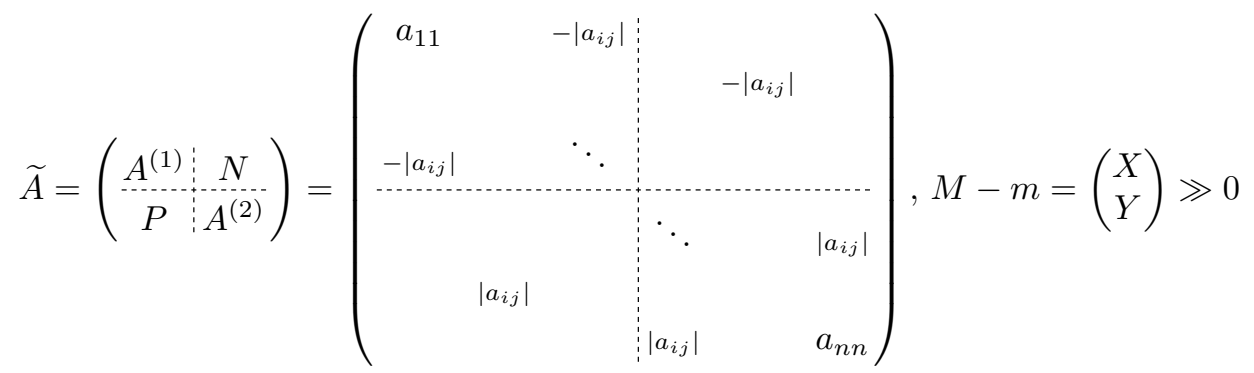

where $A^{(1)}$ is $p \times p, A^{(2)}$ is $(n-p) \times(n-p), N$ (respectively $\left.P\right)$ is a $p \times(n-p)($ resp. $(n-p) \times p)$ nonpositive (resp. nonnegative) matrix, and the dimensions of $X$ and $Y$ are respectively $p$ and $(n-p)$. Inequalities (9) and (10) then imply:

$$
\begin{cases}A^{(1)} \cdot X+N . Y & \gg 0 \\ P . X+A^{(2)} \cdot Y \ll 0\end{cases}
$$

We can now give necessary conditions for the existence of a transverse rectangle (with a symmetrical pattern) for system (5).

Theorem 1 Consider the dynamical system (5) with $A$ invertible. Let $m, M \in \mathbb{R}^{n}, 0 \ll m \ll M$, and let $R$ designate the $n$-dimensional rectangle $[m, M]$. Suppose there exists $p \in\{0, \ldots, n\}$ such that the $p$ pairs of faces $\mathcal{F}_{i}^{ \pm}$of $R(i \in\{1, \ldots, p\})$ are outgoing and the $n-p$ pairs of faces $\mathcal{F}_{i}^{ \pm}$, for $i \in\{p+1, \ldots, n\}$ are incoming. Then the two following properties hold: 
- $\left(\mathbf{P}_{\mathbf{1}}\right)$ The matrix $\mathcal{M}(A)$ is a non-singular M-matrix.

- $\left(\mathbf{P}_{\mathbf{2}}\right)$ Diagonal entries of $A$ satisfy:

$$
\begin{array}{ll}
\forall i \in\{1, \ldots, p\}, & a_{i i}>0 \\
\forall i \in\{p+1, \ldots, n\}, & a_{i i}<0
\end{array}
$$

\section{Proof}

Following our previous notations, we define the block matrix:

$$
M=\left(\begin{array}{cc}
A^{(1)} & N \\
-P & -A^{(2)}
\end{array}\right)
$$

By definition, $M$ belongs to $Z^{n \times n}$. Denoting by $\mathbf{d}$ the vector $\mathbf{d}=M-m \gg 0$, system (11) writes:

$$
M \mathbf{d} \gg 0 .
$$

which, according to [2, p 134], is equivalent to the fact that $M$ is a non-singular M-matrix. Still using this theorem, we can deduce several properties satisfied by matrix $M$. In particular, its diagonal entries are all positive, which implies, according to the definition of $M$, the property $\left(\mathbf{P}_{\mathbf{2}}\right)$. This also implies that $M$ is the comparison matrix of $A$, and so proves property $\left(\mathbf{P}_{\mathbf{1}}\right)$.

Theorem [2, p 134] provides us with several characterizations of M-matrices. For instance, one can easily show that $\mathcal{M}(A)$ is a M-matrix by checking that all its principal minors are positive. Another characterization is linked with the notion of strict diagonal dominance (see definition in $[2,9]$ ). Denoting by $D$ the diagonal matrix $D=\operatorname{diag}(\mathbf{d})$ (we recall that $\mathbf{d}$ is the positive vector $M-m)$, we have the following equivalences:

$$
\begin{aligned}
\mathcal{M}(A) \text { is a } M \text {-matrice } & \Longleftrightarrow \mathcal{M}(A) \mathbf{d} \gg 0 \\
& \Longleftrightarrow A D \text { is strictly diagonally dominant } \\
& \Longleftrightarrow \quad D^{-1} A D \text { is strictly diagonally dominant }
\end{aligned}
$$

We want now to analyse the stability of the equilibrium $x^{*}$ (that belongs to the interior of $R$ ).

Theorem 2 Under the hypothesis of theorem 1, no eigenvalue of A has a zero real part. Furthermore, A has exactly $p$ eigenvalues with a positive real part and $n-p$ eigenvalues with a negative real part.

In the case where the rectangle $R$ is positively invariant (ie if $p=0$ ), or equivalently in the case where every face of $R$ is outgoing, general theorems about M-matrices allow us to deduce directly the stability (resp. unstability) of the matrix $A$. In the general case of a symmetrical pattern with both incoming and outgoing faces, we have to come back to the more general Geršgorin theorem:

Geršgorin theorem (see notably [9, $p$ 344])

Let $M$ be a real $n \times n$ matrix. Noting, for $i \in\{1, \ldots, n\}, r_{i}(M)=\sum_{j \neq i}\left|m_{i j}\right|$ and $\mathcal{D}_{i}(M)$ the closed disc of $\mathbb{C}$ of center $m_{i i}$ and of radius $r_{i}(M)$, the eigenvalues of matrix $M$ are contained in the union of the $n$ discs: $s p(M) \subset G(M):=\bigcup_{i=1}^{n} \mathcal{D}_{i}(M)$. Furthermore, and it is the useful result here, if a union of $k$ discs forms a connected region that is disjoint from the remaining $n-k$ discs, then this region contains exactly $k$ eigenvalues of $M$. We can now give the proof of theorem 2.

\section{Proof (Theorem 2)}

We deduce from theorem 1 that the matrix $B=D^{-1} A D$ is strictly diagonally dominant. We then have, for $i \in\{1, \ldots, n\},\left|b_{i i}\right|>r_{i}(B)$. Using Geršgorin theorem, we deduce that $G(B) \cap i \mathbb{R}=$ $\varnothing$, which implies that no eigenvalue of $B$ has a zero real part. We have furthermore that, for $i \in\{1, \ldots, p\}, b_{i i}=a_{i i}>0$ and, for $i \in\{p+1, \ldots, n\}, b_{i i}=a_{i i}<0$. Using the second part of Gers̆gorin, we deduce that $B$ has exactly $p$ eigenvalues in the right complex half-plane and $n-p$ in the left complex half-plane. In order to conclude the proof of the theorem, it suffices to remark that $\operatorname{sp}(A)=\operatorname{sp}(B)$. 
We deduce from that theorem the stability of $x^{*}$ in the linear case. To analyze the stability for $\mathrm{LV}$ system, it is sufficient to remark that the jacobian matrix of the vector field $f(x)=x \otimes A\left(x-x^{*}\right)$ at the point $x^{*}$ is the matrix $\operatorname{diag}\left(x^{*}\right) A$. Given that $x^{*} \gg 0$, we deduce the following corollary:

Corollary 1 Under the hypothesis of theorem 1, the point $x^{*}$ is a hyperbolic equilibrium point for both linear and LV systems. Furthermore, it is asymptotically stable (in both cases) if and only if $p=0$ (ie $R$ has only incoming faces). Otherwise, $x^{*}$ is unstable.

\subsection{Sufficient conditions}

We are interested in this part of a converse of theorem 1. Actually, we will show that if the matrix $A$ satisfies the conclusion of theorem 1 , then it is possible to build a rectangle $R=[m, M]$ with a symmetrical pattern. We will notably see that $R$ does not have necessarily to be symmetrical (in a geometrical sense) around the equilibrium $x^{*}$, but, according to explicit constraints depending on the off-diagonal entries of $A$, it has to be "not too far" from it.

We consider system (5), with $A$ invertible. Let $p \in\{0, \ldots, n\}$, we assume that $A$ satisfies properties $\left(\mathbf{P}_{\mathbf{1}}\right)$ and $\left(\mathbf{P}_{\mathbf{2}}\right)$. Reconsidering our previous calculations, the rectangle $R=[m, M]$, with $0 \ll m \ll M$ has the wanted symmetrical transverse pattern if and only if the systems of inequations (7) and (8) are satisfied. Some calculations lead us to an equivalent system:

$$
\left\{\begin{array}{l}
a_{i i}\left(m_{i}-x_{i}^{*}\right)+\sum_{j \neq i}-\left|a_{i j}\right|\left(m_{j}-x_{j}^{*}\right)+\sum_{j \neq i} a_{i j}^{+}\left(M_{j}+m_{j}-2 x_{j}^{*}\right)<0 \\
a_{i i}\left(M_{i}-x_{i}^{*}\right)+\sum_{j \neq i}-\left|a_{i j}\right|\left(M_{j}-x_{j}^{*}\right)+\sum_{j \neq i} a_{i j}^{+}\left(M_{j}+m_{j}-2 x_{j}^{*}\right)<0 \\
a_{i i}\left(m_{i}-x_{i}^{*}\right)+\sum_{j \neq i}\left|a_{i j}\right|\left(m_{j}-x_{j}^{*}\right)+\sum_{j \neq i} a_{i j}^{-}\left(M_{j}+m_{j}-2 x_{j}^{*}\right) \\
a_{i i}\left(M_{i}-x_{i}^{*}\right)+\sum_{j \neq i}\left|a_{i j}\right|\left(M_{j}-x_{j}^{*}\right)+\sum_{j \neq i} a_{i j}^{-}\left(M_{j}+m_{j}-2 x_{j}^{*}\right)<0
\end{array}\right.
$$

Let $B$ designate the $p \times n$ upper submatrix of $A^{+}$, and $C$ designate the $(n-p) \times n$ lower submatrix of $A^{-} . B$ and $C$ are therefore respectively nonnegative and nonpositive. We also decompose the vectors $m-x^{*}, M-x^{*}, \mathbf{d}=M-m \gg 0$ and the diagonal matrix $D=\operatorname{diag}(\mathbf{d})$ into two subvectors (resp. diagonal submatrices) of respective sizes $p$ and $n-p$ :

$$
m-x^{*}=\left(\begin{array}{c}
m^{\prime} \\
m^{\prime \prime}
\end{array}\right), M-x^{*}=\left(\begin{array}{c}
M^{\prime} \\
M^{\prime \prime}
\end{array}\right), \mathbf{d}=\left(\begin{array}{l}
d^{\prime} \\
d^{\prime \prime}
\end{array}\right) \text { and } D=\left(\begin{array}{cc}
D^{\prime} & 0 \\
0 & D^{\prime \prime}
\end{array}\right)
$$

In order to build a rectangle $R=[m, M]$ that has a symmetrical pattern, we pose: $m=x^{*}+\alpha \mathbf{d}$ and $M=x^{*}+\beta \mathbf{d}$, where $\alpha$ and $\beta$ are two real numbers verifying $\alpha<\beta$. We therefore impose $m$ and $M$ to be on the affine straight line passing through $x^{*}$ and of direction $\mathbf{d}$. We finally pose $u^{\prime}=A^{(1)} d^{\prime}+N d^{\prime \prime}, u^{\prime \prime}=A^{(2)} d^{\prime \prime}+P d^{\prime}, v^{\prime}=B \mathbf{d}$ and $v^{\prime \prime}=C \mathbf{d}$. Since $\mathbf{d} \gg 0, C, N$ are nonpositive matrices and $B, P$ are nonnegative matrices, we have $v^{\prime} \geq 0$ and $v^{\prime \prime} \leq 0$. Moreover, properties $\left(\mathbf{P}_{\mathbf{1}}\right)$ and $\left(\mathbf{P}_{\mathbf{2}}\right)$ involve $u^{\prime} \gg 0$ and $u^{\prime \prime} \ll 0$. A first and direct consequence of (12) is that we have: $\alpha<0<\beta$, which implies, as we already knew, $m \ll x^{*} \ll M\left(\right.$ ie $\left.x^{*} \in R\right)$. A second consequence is the following system:

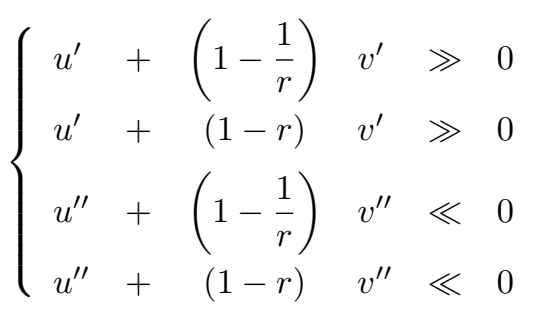

where $r$ is the ratio: $r=\left|\frac{\alpha}{\beta}\right|=-\frac{\alpha}{\beta}$. It represents the gap between $m$ and $x^{*}$ on one hand, and $M$ and $x^{*}$ on the other. Some easy calculations allow us to summarize the results of this part in the following converse of theorem 1 :

Theorem 3 Let $A$ be an invertible $n \times n$ real matrix satisfying properties $\left(\mathbf{P}_{\mathbf{1}}\right)$ and $\left(\mathbf{P}_{\mathbf{2}}\right)$. We note $\Delta$ the affine straight line: $\Delta=x^{*}+\mathbf{d} \mathbb{R}$ (d being a positive vector satisfying $\left.\mathcal{M}(A) \mathbf{d} \gg 0\right)$. If $m, M$ are two points of $\Delta$ satisfying: 
(i) $m=x^{*}+\alpha \mathbf{d}, M=x^{*}+\beta \mathbf{d}$, with $\alpha<0<\beta$, and

(ii) the ratio $r=-\frac{\alpha}{\beta}$ belongs to the interval $] \rho, \frac{1}{\rho}[$, where $\rho$ is defined by:

$$
\rho=\max \left\{\max _{i=1 \ldots p}\left(\frac{v_{i}^{\prime}}{u_{i}^{\prime}+v_{i}^{\prime}}\right), \max _{i=p+1 \ldots n}\left(\frac{v_{i}^{\prime \prime}}{u_{i}^{\prime \prime}+v_{i}^{\prime \prime}}\right)\right\} \in[0,1[
$$

then, the $n$-dimensional rectangle $R=[m, M]$ is transverse, and has a symmetrical transverse pattern with respect to system (5). More precisely, the pairs of faces $\mathcal{F}_{i}^{ \pm}$, for $i \in\{1, \ldots, p\}$, are outgoing and the pairs of faces $\mathcal{F}_{i}^{ \pm}$, for $i \in\{p+1, \ldots, n\}$, are incoming.

To give a better insight of this theorem, we make here several observations.

- First, the rectangles that we build all have the affine straight line $\Delta=x^{*}+\mathbf{d} \mathbb{R}$ (positive vector $\mathbf{d}$ being given by $\left(\mathbf{P}_{\mathbf{1}}\right)$ ) as principal diagonal (ie the lower and upper bounds $m$ and $M$ of $R$ lies on $\Delta$ ).

- As $u^{\prime}$ and $u^{\prime \prime}$ have no zero coefficients, we have $\rho<1$. Then, any rectangle $[m, M]$, with $m$ and $M$ being symmetrical relatively to $x^{*}$ is transverse (rectangle $[m, M]$ is then, in a geometrical sense, symmetrical around the equilibrium).

- In the case where matrices $B$ and $C$ are zero (ie the lines of $A$ with positive diagonal coefficient have only nonpositive off-diagonal coefficients and the lines with negative diagonal coefficient have only nonnegative off-diagonal coefficients), then $\rho=0$. Thus, any couple of points $m, M \in \Delta$ such that $m \ll x^{*} \ll M$ gives a transverse rectangle. Particularly, in the case where all the diagonal elements of $A$ are negative, this "ideal" situation occurs when $A$ is a cooperative matrix (ie every off-diagonal coefficient is nonnegative). In that case, any rectangle (of principal diagonal $\Delta$ ) containing the equilibrium is positively invariant.

- In all other cases $(0<\rho<1)$, this theorem gives explicit constraints depending on the offdiagonal terms of $A$ in order to build a transverse rectangle. The closer from 1 the constant $\rho$ is, the finer is the constraint on the choice of $m$ and $M$ (at the limit, we have only one choice: the rectangle $[m, M]$ has to be taken symmetrical around the equilibrium).

\section{Example 1}

Let us consider the three $2 \times 2$ matrices:

$$
A=\left(\begin{array}{cc}
2 & -1 \\
2 & -3
\end{array}\right), \quad A^{\prime}=\left(\begin{array}{cc}
2 & 1 \\
2 & -3
\end{array}\right), \quad A^{\prime \prime}=\left(\begin{array}{cc}
2 & 1.9 \\
2 & -3
\end{array}\right)
$$

These three matrices are strictly diagonally dominant, the vector $\mathbf{d}=\left(\begin{array}{ll}1 & 1\end{array}\right)^{t}$. We will so consider points $m$ and $M$ lying on the straight line $\Delta$ of direction $\mathbf{d}$ and passing through $x^{*}$. Given the diagonal terms of these three matrices, we are looking for a rectangle $R$ with the pattern given in fig. 4 a.

- The matrix $A$ is the "ideal" case, where $B=C=0$. Then $v^{\prime}=v^{\prime \prime}=0$ and $\rho=0$. Any couple of points $m, M \in \Delta$ such that $m \ll x^{*} \ll M$ gives a rectangle $R$ that is transverse and has the pattern of fig $4 a$.

- For $A^{\prime}$, we have $\rho=\frac{1}{2}=0.5$,

- and for $A^{\prime \prime}, \rho=\frac{1.9}{2}=0.95$.

The figure $4 b$ represents the constraints in the cases of $A^{\prime}$ and $A^{\prime \prime}$. We see on this figure that the higher is the positive term $a_{12}$, the finer is the constraint on the size of the rectangle $R=[m, M]$, and the closer $R$ must be to the rectangle symmetrical around the equilibrium. 


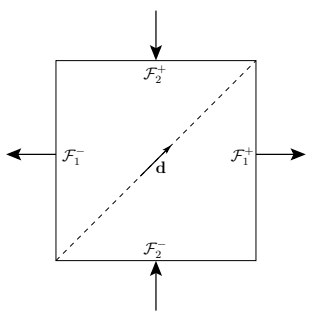

(a)
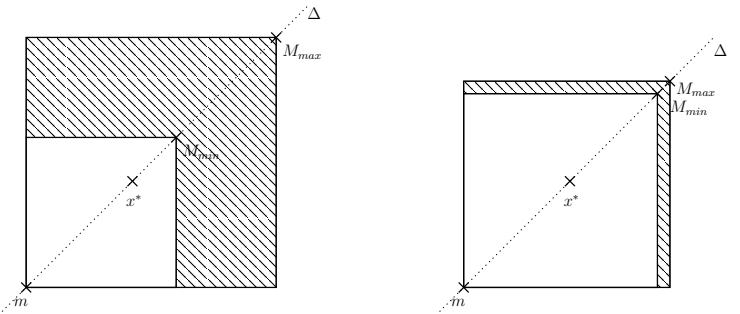

(b)

Figure 4: (a): Transverse pattern for matrices $A, A^{\prime}$ and $A^{\prime \prime}$. (b): The point $m$ being fixed, we represent the range for $M$ imposed by theorem 3 in order to have a rectangle $[m, M]$ with the pattern of figure $4 \mathrm{a}$ (on the left: $A^{\prime}$, on the right: $A^{\prime \prime}$ ).

\section{Dynamical behavior within transverse rectangles}

We propose in this part to analyze the dynamical behavior of systems (1) and (2) within a transverse rectangle, with a symmetrical pattern. Thanks to theorem 2 and its corollary, we know the local behavior of these systems in a neighborhood of their equilibrium $x^{*}$. We want now to extend the analysis of their behavior within the whole rectangle. The main result of this part lies in theorem 4. This theorem ensures that, as soon as there exists a transverse rectangle containing the equilibrium point $x^{*}$ (and the precedent part gives necessary and sufficient conditions, together with a method to find such rectangles), this rectangle is either contracting towards the equilibrium, or left in finite time (ie it is transient). In particular, such a rectangle cannot contain an omega-limit set different from $\left\{x^{*}\right\}$ (it cannot contain a limit cycle for instance). This result is straightforward in the case of linear systems but not in the Lotka-Volterra framework in which, as we already said, complex asymptotic behavior may occur in dimension $n \geq 3$.

This part is mainly based on Lyapunov theory, notably on stability and instability theorem, that can be found for instance in [10]. The Lyapunov candidate functions that we use are based on vector norms (see notably $[8,13]$ ).

We assume the existence of a rectangle $R=[m, M]$ and of $p \in\{0, \ldots, n\}$ such that the $p$ pairs of faces $\mathcal{F}_{i}^{ \pm}, i=1, \ldots, p$ of $R$ are outgoing and the $n-p$ pairs of faces $\mathcal{F}_{i}^{ \pm}, i=p+1, \ldots, n$ are incoming. Following our previous notations, we denote by $\mathbf{d}$ the vector $\mathbf{d}=M-m \gg 0$. According to previous parts, we know that $x^{*}$ belongs to the interior of $R$ and that matrix $A$ satisfies properties $\left(\mathbf{P}_{\mathbf{1}}\right)$ and $\left(\mathbf{P}_{\mathbf{2}}\right)$. Equivalently, it is clear that $A$ satisfies these properties if $A^{t}$ does. So there exists a vector $\mathbf{g} \gg 0$ such that:

$$
\mathcal{M}\left(A^{t}\right) \mathbf{g}=\mathcal{M}(A)^{t} \mathbf{g} \gg 0
$$

We then pose:

$$
\pi:=\min _{i=1 \ldots n}\left(\left|a_{i i}\right|-\frac{1}{g_{i}} \sum_{j \neq i}\left|a_{j i}\right| g_{j}\right)
$$

which is a positive constant. We define the vector $\widetilde{\mathbf{g}}$ as follows:

$$
\forall i \in\{1, \ldots, n\}, \widetilde{g}_{i}= \begin{cases}-g_{i} & \text { if } a_{i i}>0 \\ g_{i} & \text { if } a_{i i}<0\end{cases}
$$

We will now define a function which will be shown to be a candidate Lyapunov function for system (1). To do so, we adopt the notations of [13, p 236] and define the functional $\sigma_{i}$ as

$$
\sigma_{i}= \begin{cases}1 & \text { if } x_{i}(t)>x_{i}^{*} \text { or if } x_{i}(t)=x_{i}^{*} \text { and } \dot{x}_{i}(t)>0 \\ 0 & \text { if } x(t)=x^{*} \\ -1 & \text { if } x_{i}(t)<x_{i}^{*} \text { or if } x_{i}(t)=x_{i}^{*} \text { and } \dot{x}_{i}(t)<0\end{cases}
$$


We define now the function $V: R \rightarrow \mathbb{R}$ :

$$
V(x)=\sum_{i=1}^{n} \widetilde{g}_{i}\left|\ln \left(x_{i}\right)-\ln \left(x_{i}^{*}\right)\right|=\widetilde{\mathbf{g}}^{t} \cdot\left|\ln (x)-\ln \left(x^{*}\right)\right|
$$

Proposition 3 Function $V$ is continuous, piecewise $\mathcal{C}^{1}$ on $\left(\mathbb{R}_{+}^{*}\right)^{n}$. Furthermore, the right-hand time derivative ${ }^{3}$ of $V$ with respect to (1), noted $D^{+} V$, satisfies $D^{+} V\left(x^{*}\right)=0$ and:

$$
\forall x \in R, D^{+} V(x) \leq-\pi \mathbf{g}^{t}\left|x-x^{*}\right|
$$

Proof

The first point is straightforward, let us prove inequation (15).

Let $x \in R \backslash\left\{x^{*}\right\}$. Using (13), we have:

$$
\begin{aligned}
D^{+} V(x) & =\sum_{i=1}^{n} \widetilde{g}_{i} \sigma_{i} A_{i} \cdot\left(x-x^{*}\right) \\
& =\sum_{i=1}^{n} \sum_{j=1}^{n} \widetilde{g}_{i} \sigma_{i} \sigma_{j} a_{i j}\left(\sigma_{j}\left(x_{j}-x_{j}^{*}\right)\right)
\end{aligned}
$$

According to the definition of the functionals $\sigma$, we have $\sigma_{j}\left(x_{j}-x_{j}^{*}\right)=\left|x_{j}-x_{j}^{*}\right|$. Let us pose: $B=\left(b_{i j}\right)_{i, j}=\left(\sigma_{i} \sigma_{j} a_{j i}\right)_{i, j} \in \mathbb{R}^{n \times n}$. We remark that $B$ and $A$ have the same diagonal entries and that, for all $i \neq j,\left|b_{i j}\right|=\left|a_{i j}\right|$. We obtain: $D^{+} V(x)=\widetilde{\mathbf{g}}^{t} \cdot B^{t} .\left|x-x^{*}\right|$, with:

$$
\begin{aligned}
\left(\widetilde{g}^{t} B\right)_{i}=\sum_{j=1}^{n} \widetilde{g}_{j} b_{j i} & =\widetilde{g}_{i} a_{i i}+\sum_{j \neq i} \widetilde{g}_{j} b_{j i} \\
& \leq-g_{i}\left|a_{i i}\right|+\sum_{j \neq i} g_{j}\left|a_{j i}\right| \\
& \leq-g_{i} \pi
\end{aligned}
$$

which implies $D^{+} V(x) \leq-\pi \mathbf{g}^{t}\left|x-x^{*}\right|$.

It is easy to see (using (14)) that if $p=0$ (ie if rectangle $R$ has no outgoing faces), then $V$ is positive definite, which implies that $V$ is a Lyapunov function for system (1) (see figure 5 for an illustration when $n=2$ ). It is easy to see that in this case, $V$ is radially unbounded, which involves that $x^{*}$ is globally asymptotically stable in $\left(\mathbb{R}_{+}^{*}\right)^{n}$. If $p=0$, the rectangle $R$ is so contracting to the equilibrium $x^{*}$. In the opposite case, if $p=n$ ( $R$ has no incoming faces), then $V$ is negative definite and $-V$ is a Lyapunov function for the system in reversed time. We then deduce that, apart from the equilibrium itself, any trajectory leaves $R$ in finite time (actually it leaves any compact of $\left.\left(\mathbb{R}_{+}^{*}\right)^{n}\right)$.

The interesting case is intermediate, where $0<p<n$ ( $R$ has both incoming and outgoing faces). In that case $V$ can take both negative and positive values (see figure and 5 ). We define the sets:

$$
\left\{\begin{array}{l}
R^{-}=R \cap\left\{x \in \mathbb{R}^{n} \mid V(x)<0\right\} \\
R^{+}=R \cap\left\{x \in \mathbb{R}^{n} \mid V(x) \geq 0\right\}
\end{array}\right.
$$

Lemma 3 Let $x^{0} \in R^{-}$. For all time $t \geq 0$ such that $\left\{x\left(\tau, x^{0}\right) \mid 0 \leq \tau \leq t\right\}$ is contained in $R^{-}$, we have: $V\left(x\left(t ; x^{0}\right)\right) \leq c_{1} t+c_{2}$, where $c_{1}$ and $c_{2}$ are negative constants.

\section{Proof}

Let $x^{0} \in\left(\mathbb{R}_{+}^{*}\right)^{n}$ such that $V\left(x^{0}\right)=a<0$ and let $x(t)$ denote the solution of system (1) with initial condition $x(0)=x^{0}$. Let $t \geq 0$ be such that $\{x(\tau) \mid 0 \leq \tau \leq t\} \subset R^{-}$. According to the precedent

\footnotetext{
${ }^{3}$ The derivative is in the sense of Dini: $D^{+} f(t)=\limsup _{h \rightarrow 0^{+}} \frac{1}{h}[f(t+h)-f(t)]$
} 
proposition, $D^{+} V$ is nonpositive, implying that, for all $0 \leq \tau \leq t, V(x(\tau)) \leq a<0$. Using the definition of $V$, one can write, for all $0 \leq \tau \leq t$ :

$$
V(x(\tau))=-\sum_{i=1}^{p} g_{i}\left|\ln \left(x_{i}(\tau)\right)-\ln \left(x_{i}^{*}\right)\right|+\sum_{i=p+1}^{n} g_{i}\left|\ln \left(x_{i}(\tau)\right)-\ln \left(x_{i}^{*}\right)\right| \leq a
$$

implying $\mathbf{g}^{t}\left|\ln (x(\tau))-\ln \left(x^{*}\right)\right| \geq-a>0$. We now use the fact that $x(\tau)$ belongs to the rectangle $R$. Thanks to the mean-value theorem:

$$
\forall i \in\{1, \ldots, n\}, \forall x_{i} \in\left[m_{i}, M_{i}\right],\left|\frac{\ln \left(x_{i}\right)-\ln \left(x_{i}^{*}\right)}{x_{i}-x_{i}^{*}}\right| \leq \frac{1}{m_{i}} \leq \frac{1}{m^{*}}
$$

where $m^{*}=\min _{i=1 \ldots n} m_{i}>0$. To complete the proof, we finally write (using (15)):

$$
\begin{aligned}
V(x(t)) & =V\left(x^{0}\right)+\int_{0}^{t} D^{+} V(x(\tau)) d \tau \\
& \leq a-\pi \int_{0}^{t} \mathbf{g}^{t}\left|x(\tau)-x^{*}\right| d \tau \\
& \leq a-\pi m^{*} \int_{0}^{t} \mathbf{g}^{t}\left|\ln (x(\tau))-\ln \left(x^{*}\right)\right| d \tau \\
& \leq c_{2}+c_{1} t
\end{aligned}
$$

where $c_{1}=\pi m^{*} a$ and $c_{2}=a$ are negative constants.

We can now give the following theorem, describing the dynamical behavior of system (1) in the transverse rectangle $R$.

Theorem 4 Consider dynamical system (1) in $\left(\mathbb{R}_{+}^{*}\right)^{n}$. Let $m, M \in\left(\mathbb{R}_{+}^{*}\right)^{n}$ such that $m \ll M$ and such that the rectangle $R=[m, M]$ has $p \in\{0, \ldots, n\}$ pairs of outgoing faces $\mathcal{F}_{i}^{ \pm}, i=1, \ldots, p$ and $n-p$ pairs of incoming faces $\mathcal{F}_{i}^{ \pm}, i=p+1, \ldots, n$. Then,

- if $p=0$, any trajectory starting in $R$ converges towards the equilibrium $x^{*}$.

- if $p=n$, any trajectory starting in $R$ (except the equilibrium itself) leaves $R$ in finite time.

- If $1 \leq p \leq n-1$, then for almost every initial condition $x^{0} \in R$, the solution $x\left(t ; x^{0}\right), t \geq 0$ of (1) leaves $R$ in finite time.

\section{Proof}

We prove here only the third point, as the other ones are direct consequences of Lyapunov's stability theorems. The following proof is inspired from the proof of Chetaev instability theorem (see $[10, \mathrm{p} 113])$.

When $0<p<n-1, R^{+} \neq \varnothing, R^{-} \neq \varnothing$, and $\left\{R^{+}, R^{-}\right\}$forms a partition of $R$. We first show that for any initial condition $x^{0} \in R^{-}$, the solution $x\left(t ; x^{0}\right)$ leaves $R$ in finite time. Let $x^{0} \in R^{-}$, such that $V\left(x^{0}\right)=a<0$. According to lemma 3 , as long as $x\left(t, x^{0}\right)$ stays in $R^{-}$, we have $V\left(x\left(t ; x^{0}\right)\right) \leq c_{1} t+c_{2}$. As $V$ is continuous on $\left(\mathbb{R}_{+}^{*}\right)^{n}$ and $R^{-}$is bounded, this implies that $x\left(t ; x^{0}\right)$ cannot stay in $R^{-}$for all times. As it cannot leave $R^{-}$through the surface $V(x)=0$, it has to leave $R$ in finite time.

Let us now consider the case when $x^{0} \in R^{+}$. We will use LaSalle theorem (see for instance [12]) to prove that almost all such $x^{0}$ leaves $R$ in finite time. Let us distinguish the two possible cases:

- Suppose that the whole trajectory lies in $R^{+}$, ie that $\left\{x\left(t ; x^{0}\right) \mid t \geq 0\right\} \subset R^{+}$. According to LaSalle, this implies that $x\left(t ; x^{0}\right)$ converges to the largest positively invariant set contained in $\left\{x \mid D^{+} V(x)=0\right\}$. In our case, as $D^{+} V$ is negative definite, this yields to: $x\left(t ; x^{0}\right) \underset{t \rightarrow+\infty}{\longrightarrow} x^{*}$. According to theorem 2, this means that $x^{0}$ lies in the stable manifold of $x^{*}$, which is of dimension $n-p<n$ (it is therefore of Lebesgue measure zero). 
- The second case is when $x\left(t ; x^{0}\right)$ leaves $R^{+}$in finite time, which means that either it directly leaves $R$, or it stays in $R$ and reaches $R^{-}$(and we are brought back to the previous case).

In conclusion, if we except the stable manifold of $x^{*}$ which is of measure zero, for any initial condition $x^{0} \in R$, the solution $x\left(t ; x^{0}\right)$ leaves $R$ in finite time.
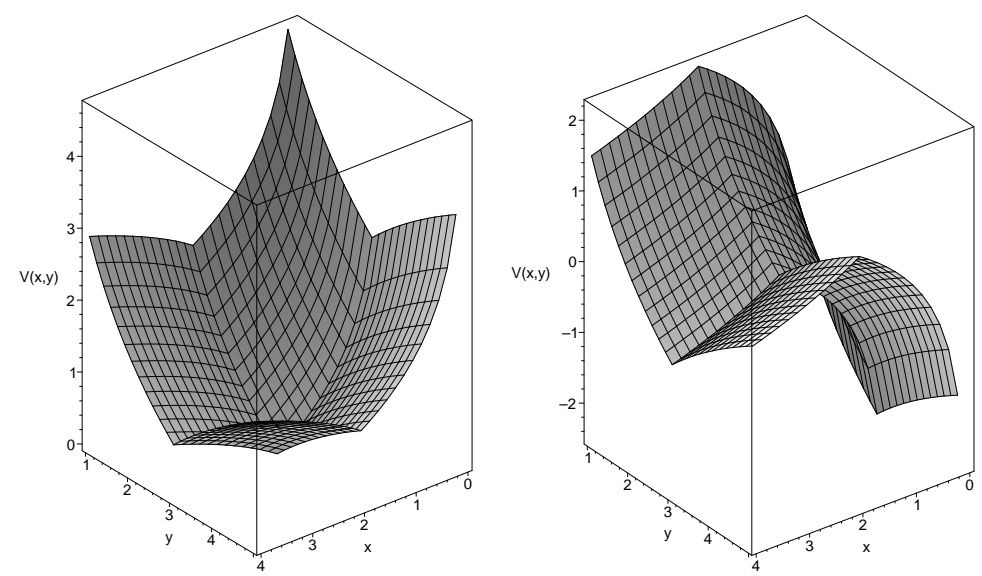

Figure 5: Representation of function $V$ for 2-dimensional system with $x^{*}=(2,3)$ and $\mathbf{g}=(1,2)$. On the left: $p=0$ ( $V$ is a Lyapunov function), on the right: $p=1$.

\section{Conclusion}

Theoretical results presented here set up a first step towards a qualitative abstraction of LotkaVolterra dynamical systems. Thanks to proposition 2 and theorem 4 , we classify the different dynamical behaviors of a LV system within rectangles that either contain the equilibrium or not. If $A$ does not satisfy property $\left(\mathbf{P}_{\mathbf{1}}\right)$, we have proved that it is impossible to build a transverse rectangle around the equilibrium. A possible extension is to study the hybrid behavior of a LV system on a rectangular mesh. We should then focus on the control of the system on rectangles (in order for instance to steer the trajectories to a designated face). As an example, the linear feedback approach presented in [7] can be extended in the LV framework. Another extension is the study of a general Lotka-Volterra hybrid system, with different LV systems in each rectangle; as in [4], the vector field then becomes discontinuous, and we have to face complex issues such as sliding motions and Filippov solutions on the boundaries.

\section{References}

[1] A. Abate and A. Tiwari. Box invariance of hybrid and switched systems. In IFAC Conf. on Analysis and Design of Hybrid Systems, 2006.

[2] A. Berman and R.J. Plemmons. Nonnegative matrices in the mathematical sciences. Classics in Applied Mathematics. SIAM Press, 1994.

[3] E.B. Castelan and J.C. Hennet. On invariant polyhedra of continuous-time linear systems. IEEE Transactions on Automatic control, 38(11):1680-1685, 1993.

[4] H. de Jong, J.-L. Gouzé, C. Hernandez, M. Page, T. Sari, and J. Geiselmann. Qualitative simulation of genetic regulatory networks using piecewise-linear models. Bull. Math. Biol., 66:301-340, 2005.

[5] L. Farina and L. Benvenuti. Invariant polytopes of linear systems. IMA Journal of Mathematical Control and Information, 15:233-240, 1998. 
[6] J.-L. Gouzé. Positivity, space scale, and convergence towards the equilibrium. Journal of Biological Systems, 3(2):613-620, 1995.

[7] L.C.G.J.M. Habets and J.H. van Schuppen. A control problem for affine dynamical systems on a full-dimensional polytope. Automatica, 40:21-35, 2004.

[8] J. Hofbauer and K. Sigmund. Evolutionary games and population dynamics. Cambridge University Press, 1998.

[9] R.A. Horn and C.R. Johnson. Matrix analysis. Cambridge University Press, 1999.

[10] H.K. Khalil. Nonlinear systems. Upper Saddle River, NJ: Prentice Hall, 2002.

[11] M. Kloetzer and C. Belta. Reachability analysis of multi-affine systems. In Hybrid Systems: Computation and Control. Springer, 2006.

[12] N. Rouche and J. Mawhin. Equation différentielles ordinaires. Masson et cie, 1973.

[13] D.D. Šiljak. Large-scale dynamic systems, stability and structure. North-Holland series in System Science and Engineering. Elsevier, North-Holland, 1978.

[14] G.M. Ziegler. Lectures on polytopes. Graduate Texts in Mathematics. Springer-Verlag, NewYork, 1995. 


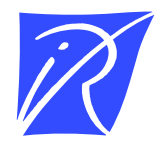

\section{Unité de recherche INRIA Sophia Antipolis 2004, route des Lucioles - BP 93 - 06902 Sophia Antipolis Cedex (France)}

Unité de recherche INRIA Futurs : Parc Club Orsay Université - ZAC des Vignes 4, rue Jacques Monod - 91893 ORSAY Cedex (France)

Unité de recherche INRIA Lorraine : LORIA, Technopôle de Nancy-Brabois - Campus scientifique 615, rue du Jardin Botanique - BP 101 - 54602 Villers-lès-Nancy Cedex (France)

Unité de recherche INRIA Rennes : IRISA, Campus universitaire de Beaulieu - 35042 Rennes Cedex (France)

Unité de recherche INRIA Rhône-Alpes : 655, avenue de l'Europe - 38334 Montbonnot Saint-Ismier (France)

Unité de recherche INRIA Rocquencourt : Domaine de Voluceau - Rocquencourt - BP 105 - 78153 Le Chesnay Cedex (France) 